\title{
Quark ACM with topologically generated gluon mass
}

\section{Ishita Dutta Choudhury*}

S N Bose National Centre for Basic Sciences, Salt Lake, Kolkata, India

E-mail: ishitadutta. choudhuryabose.res.in

\section{Amitabha Lahiri}

S N Bose National Centre for Basic Sciences, Salt Lake, Kolakata-700098, India

\begin{abstract}
We investigate the effect of a small gauge-invariant mass of the gluon, on the anomalous chromomagnetic moment (ACM) of quarks, by perturbative calculations at one loop level. The gluon is taken to acquire its mass through a topological mass generation mechanism, via an interaction with an antisymmetric tensor field $B_{\mu \nu}$. For a gluon mass between 0 and $10 \mathrm{MeV}$, we calculate the $\mathrm{ACM}$ at momentum transfer $q^{2}=-M_{Z}^{2}$. These are compared with the ACM when the gluon mass arises from a Proca term. We find that the ACM of up, down, strange, and charm vary significantly with gluon mass, while the ACM of top and bottom quarks show negligible variation. The mechanism of gluon mass generation is most important for the strange quark, but not so much for the other quarks.
\end{abstract}

The European Physical Society Conference on High Energy Physics

22-29 July 2015

Vienna, Austria

${ }^{*}$ Speaker. 


\section{Introduction}

The mass of the gluon is theoretically taken to be zero in QCD, the theory of strong interactions. Experimentally however, the mass of the gluon is yet to be determined with sufficient precision. Previous analyses regarding this quantity provide a large range of values, starting from $O(1) \mathrm{MeV}$ to a few hundred $\mathrm{MeV}$, for a possible gluon mass $[1,2,3,4,5,6]$. A more stringent bound on gluon mass may become available from precision experiments in QCD in the near future, as the Linear Hadron Collider reaches new peaks of luminosity and energy, providing us with a new arena of precision QCD. The mass of the gluon cannot come from a Higgs mechanism however, since color symmetry is known to be unbroken. There are some mechanisms which can produce a mass for a gauge boson without symmetry breaking [1,7]. The topological mass generation mechanism $[8,9,10]$ is one of them.

Precision experiments in QCD will also be able to investigate the Anomalous Chromomagnetic Moment (ACM) of quarks. This quantity has received negligible attention from the community so far, as there has been only one experimental analysis of the top quark ACM based on the LHC data [11]. At low energies, the ACM is defined only in non-perturbative $\mathrm{QCD}$, but it is possible to calculate it perturbatively at high energies, where the QCD coupling is small. In this paper, we present perturbative calculation of the anomalous chromomagnetic moment of quarks, and because it would be subject to precision measurements, we also assume a small, topologically generated, gauge-invariant mass for the gluon.

We work with the Lagrangian

$$
L=-\frac{1}{4} F_{\mu \nu}^{a} F_{a}^{\mu v}+\bar{\psi}\left(i \gamma^{\mu} \partial_{\mu}-m\right) \psi+j_{a}^{\mu} G_{\mu}^{a}+\frac{1}{12} H_{a}^{\mu \nu \lambda} H_{\mu \nu \lambda}^{a}+\frac{M}{4} \varepsilon^{\mu \nu \rho \lambda} F_{a}^{\mu v} B_{\rho \lambda}^{a},
$$

where $F_{\mu \nu}^{a}$ is the field strength tensor of the gluon field $G^{\mu a}, H_{\mu \nu \lambda}^{a}=\partial_{[\mu} B_{\rho \lambda]}^{a}-g f^{a b c} G_{[\mu}^{b} B_{\rho \lambda]}^{c}$ is the field strength tensor of the antisymmetric tensor field $B_{\mu \nu}^{a}, M$ is the gluon mass, $m$ is the quark mass, and

$$
j_{\mu}^{a}=g \bar{\psi} T^{a}\left[\gamma_{\mu}+i F_{2}\left(q^{2}\right) \sigma_{\mu \nu} q^{v}+\cdots\right] \psi
$$

is the effective current including quantum corrections, with $T^{a}$ the generators of SU(3). The coefficient $F_{2}\left(q^{2}\right)$ is said to be the anomalous chromomagnetic moment (ACM) of the quark at the momentum transfer $q$, this is what we will calculate at one loop in perturbation theory. This Lagrangian is invariant under the local $S U(3)$ gauge transformations

$$
\begin{aligned}
G_{\mu}^{a} & \rightarrow U G_{\mu}^{a} U^{-1}-\frac{i}{g} \partial_{\mu} U U^{-1}, \\
B_{\mu \nu}^{a} & \rightarrow U B_{\mu \nu}^{a} U^{-1} .
\end{aligned}
$$

In order to get the propagators, we add two 'gauge fixing terms' to the Lagrangian. These are

$$
\mathscr{L}_{G F}=-\frac{1}{2 \xi}\left(\partial_{\mu} G_{a}^{\mu}\right)^{2}-\frac{1}{2 \eta}\left(D_{\mu} B_{a}^{\mu v}\right)^{2}
$$

where $\xi$ and $\eta$ are the gauge fixing parameters for the gluon and the antisymmetric tensor field $B_{\mu \nu}^{a}$ respectively. The propagators can now be calculated,

$$
i \Delta^{\mu v, a b}=-\frac{i}{k^{2}-M^{2}}\left(g^{\mu v}-(1-\xi) \frac{k^{\mu} k^{v}}{k^{2}}\right) \delta^{a b},
$$




$$
i \Delta^{\mu \nu, \rho \lambda ; a b}=\frac{i}{k^{2}}\left(g^{\mu[\rho} g^{\lambda] v}-(1-\eta) \frac{k^{\mu} k^{[\lambda} g^{\rho] v}-k^{v} k^{[\lambda} g^{\rho] \mu}}{k^{2}}\right) \delta^{a b} .
$$

The $B F$ interaction term contains a two-point vertex, which corresponds to an off-diagonal mixing term between the gluon and the $B$ fields. Inserting the $B$ propagator via the 2-point vertex as in Fig. 1, we find the 'effective' bare propagator of the gluon field,

$$
i D_{\mu v}=-i \frac{g_{\mu v}-k_{\mu} k_{v} / k^{2}}{k^{2}-M^{2}+i \varepsilon}+i \xi \frac{k_{\mu} k_{v}}{k^{4}} .
$$

The $k_{\mu} k_{v}$ terms do not contribute to the ACM at one loop, since the gluon couples to a conserved charged current at least at one end. The ACM of quarks receives contributions from both strong and

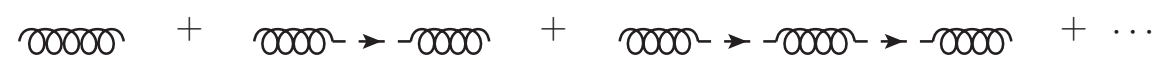

Figure 1: Bare gluon propagator by summing over all possible insertions of the $B$ propagator

weak processes at one loop order. The diagrams corresponding to strong and weak contributions are shown in Fig. 2. There are additional diagrams due to the three point vertices coming from

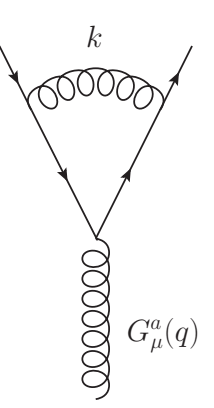

(a)

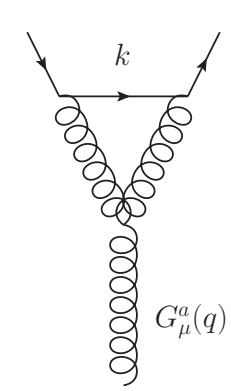

(b)

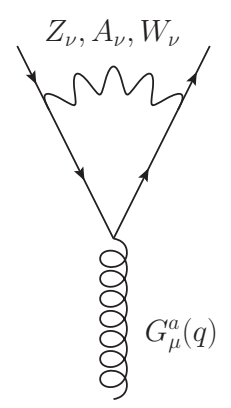

(c)

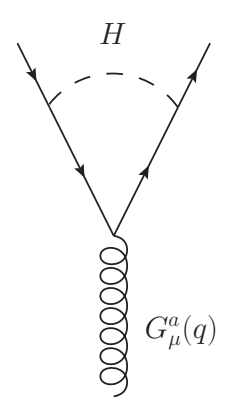

(d)

Figure 2: Contributions to the ACM of a quark: strong: (a) QED-like diagram; (b) purely non-Abelian contribution; electroweak: (c) gauge boson exchange; (d) Higgs boson exchange.

the topological mass mechanism. These new diagrams are shown in Fig. 3. Since the $B$-field does not couple to quarks directly, these diagrams can be thought of as a correction to the diagram in Fig. 2(b).

\section{Calculations}

The contribution to the ACM of quarks from the diagrams in Fig. 3 can be calculated to be

$$
\begin{aligned}
F_{2}^{T M}\left(q^{2}=-M_{Z}^{2}\right)=\frac{M^{2} \alpha_{s}}{m^{2} \pi}\left[2 \int_{0}^{1} d \zeta_{1} \int_{0}^{1-\zeta_{1}} d \zeta_{2} \int_{0}^{1-\zeta_{1}-\zeta_{2}} d \zeta_{3} \frac{\zeta_{1}\left(\frac{9}{4} \zeta_{1}+\zeta_{3}\right)}{\left[\zeta_{1}^{2}+\zeta_{3}\left(1-\zeta_{1}-\zeta_{3}\right) \frac{M_{Z}^{2}}{m^{2}}+\left(\zeta_{2}+\zeta_{3}\right) \frac{M^{2}}{m^{2}}\right]^{2}}\right. \\
-\int_{0}^{1} d \zeta_{1} \int_{0}^{1-\zeta_{1}} d \zeta_{2} \int_{0}^{1-\zeta_{1}-\zeta_{2}} d \zeta_{3} \int_{0}^{1-\zeta_{1}-\zeta_{2}-\zeta_{3}} d \zeta_{4}
\end{aligned}
$$




$$
\begin{aligned}
& \times \frac{\left.\zeta_{1}\left(1-2 \zeta_{1}\right)\left\{\zeta_{1}^{2}+\left(\zeta_{2}+\zeta_{3}\right)\left(1-\zeta_{1}-\zeta_{2}-\zeta_{3}\right) \frac{M_{Z}^{2}}{m^{2}}-\zeta_{1}^{3} \frac{M_{Z}^{2}}{m^{2}}+3 \zeta_{1}\left(\zeta_{2}+\zeta_{4}\right)\right\}\right)}{\left[\zeta_{1}^{2}+\left(\zeta_{2}+\zeta_{3}\right)\left(1-\zeta_{1}-\zeta_{2}-\zeta_{3}\right) \frac{M_{Z}^{2}}{m^{2}}+\left(\zeta_{2}+\zeta_{4}\right) \frac{M^{2}}{m^{2}}\right]^{3}} \\
& \left.-\frac{9 M_{Z}^{2}}{2 m^{2}} \int_{0}^{1} d \zeta_{1} \int_{0}^{1-\zeta_{1}} d \zeta_{2} \frac{\zeta_{1}}{\zeta_{1}^{2}+\zeta_{2}\left(1-\zeta_{1}-\zeta_{2}\right) \frac{M_{Z}^{2}}{m^{2}}+\left(1-\zeta_{1}\right) \frac{M^{2}}{m^{2}}}\right]
\end{aligned}
$$

Similarly, the strong contributions to the ACM of quarks are calculated from the diagrams in

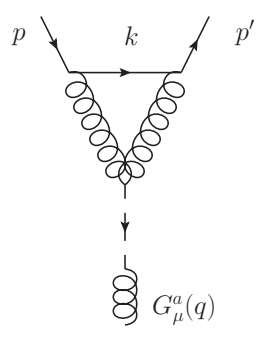

(a)

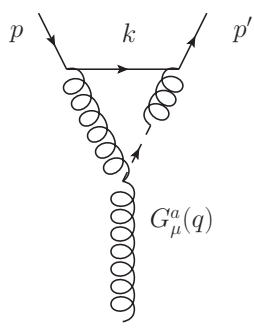

(b)

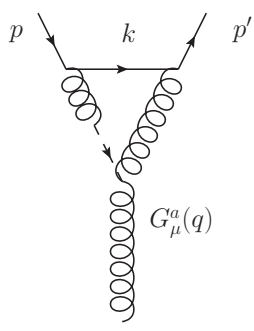

(c)

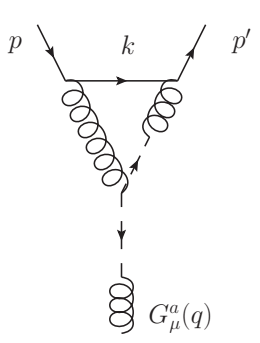

(d)

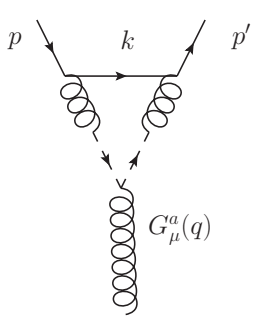

(e)

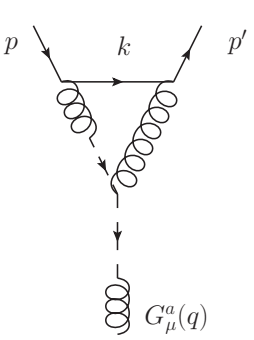

(f)

Figure 3: Topological contribution to the ACM of a quark.

Fig. 2(a) and Fig. 2(b) to be

$$
F_{2}^{(2(a))}\left(q^{2}=-M_{Z}^{2}\right)=-\frac{\alpha_{s}}{12 \pi m} \int_{0}^{1} d \zeta_{3} \int_{0}^{1-\zeta_{3}} d \zeta_{2} \frac{\left(1-\zeta_{3}\right) \zeta_{3}}{\left(1-\zeta_{3}\right)^{2}+\left(1-\zeta_{2}-\zeta_{3}\right) \zeta_{2}\left(\frac{M_{Z}}{m}\right)^{2}+\zeta_{3}\left(\frac{M}{m}\right)^{2}},
$$

and

$$
F_{2}^{(2(b))}\left(q^{2}=-M_{Z}^{2}\right)=\frac{\alpha_{s}}{8 \pi m} \int_{0}^{1} d \zeta_{3} \int_{0}^{1-\zeta_{3}} d \zeta_{2} \frac{\left(1-\zeta_{3}\right) \zeta_{3}}{\zeta_{3}^{2}+\left(1-\zeta_{2}-\zeta_{3}\right) \zeta_{2}\left(\frac{M_{Z}}{m}\right)^{2}+\left(1-\zeta_{3}\right)\left(\frac{M}{m}\right)^{2}} .
$$

We need to add these contributions to ACM together with weak contributions coming from the diagrams in Fig. 2(d). The weak contributions to the dimensionless quantity $4 m F_{2}\left(q^{2}=-M_{z}^{2}\right)$ are shown in Table 1 for each quark. In this table, a zero indicates that the corresponding entry is smaller by at least a factor of $10^{-3}$ than the largest number in the same row. Adding all the contributions, we plot the total value of $4 m F_{2}\left(q^{2}=-M_{z}^{2}\right)$ against gluon mass between 0 to 10 $\mathrm{MeV}$. For each quark, this is shown as a solid line in each of the plots in Fig. 4. For comparison, we plot the same quantity for each quark when the gluon mass is taken to come from a Proca term, as was calculated in [13]. These are represented as dotted lines in the plots in Fig. 4. In our calculations the numerical values for the physical parameters are taken from [14]. 


\begin{tabular}{cccccc}
\hline \hline Quark & $Z$ & $A$ & $W$ & $H$ & Total \\
\hline $\mathrm{u}$ & $1.29 \times 10^{-12}$ & $29.79 \times 10^{-12}$ & $-4.41 \times 10^{-12}$ & 0 & $26.67 \times 10^{-12}$ \\
$\mathrm{~d}$ & $5.50 \times 10^{-12}$ & $30.18 \times 10^{-12}$ & $-4.41 \times 10^{-12}$ & 0 & $31.27 \times 10^{-12}$ \\
$\mathrm{c}$ & $3.98 \times 10^{-7}$ & $36.91 \times 10^{-7}$ & $-0.48 \times 10^{-7}$ & 0 & $40.41 \times 10^{-7}$ \\
$\mathrm{~s}$ & $0.22 \times 10^{-8}$ & $0.82 \times 10^{-8}$ & $-4.82 \times 10^{-8}$ & 0 & $-3.78 \times 10^{-8}$ \\
$\mathrm{t}$ & $25.66 \times 10^{-4}$ & $10.57 \times 10^{-4}$ & $-2.87 \times 10^{-4}$ & $149.91 \times 10^{-4}$ & $183.27 \times 10^{-4}$ \\
$\mathrm{~b}$ & $4.16 \times 10^{-6}$ & $7.14 \times 10^{-6}$ & $-98.72 \times 10^{-6}$ & $0.03 \times 10^{-6}$ & $-87.39 \times 10^{-6}$ \\
\hline
\end{tabular}

Table 1: Electroweak contribution to $4 m F_{2}\left(q^{2}=-M_{Z}^{2}\right)$ of each quark

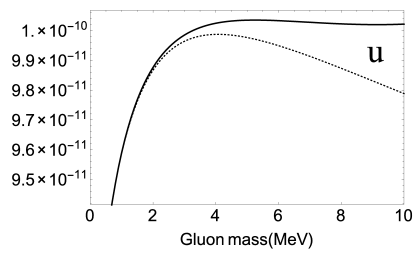

(a)

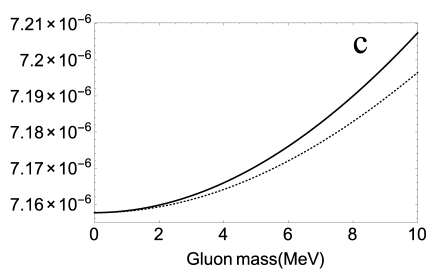

(d)

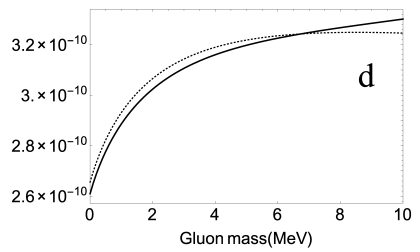

(b)

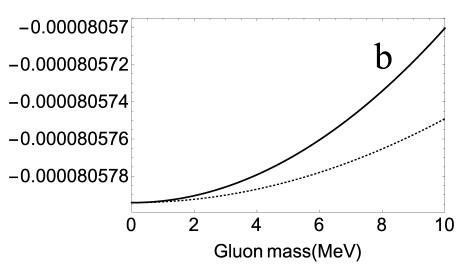

(e)

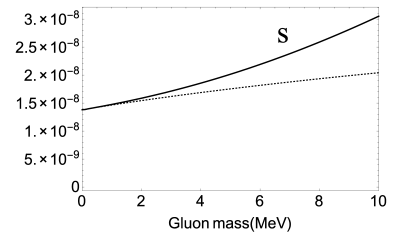

(c)

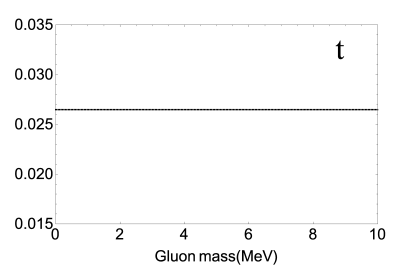

(f)

Figure 4: $4 m F_{2}$ of quarks at $q^{2}=-M_{Z}^{2}$; continuous lines represent dependence on topologically generated gluon mass; dotted lines represent dependence on gluon mass coming from a Proca term.

\section{Results and Discussions}

The ACM of quarks were calculated in [13] considering a small Proca mass for the gluon. The calculations there are relevant for the Curci-Ferrari model [7] or the dynamical mass generation mechanism $[1,2]$ for the gluon. In this talk, we have discussed the effect of a small, topologically generated gluon mass on quark $\mathrm{ACM}$ at $q^{2}=-M_{z}^{2}$. It turns out that the gluon mass dependence of the ACM is most prominent for the light quarks, and not so much for the heavy quarks. For the $s$ quark, the mechanism is also important - as the gluon mass is increased from 0 to $10 \mathrm{MeV}$, the dimensionless quantity $4 m F_{2}$ varies by more than $70 \%$ when the gluon mass is topologically generated, while for a Proca mass this variation is about $42 \%$. For other quarks, any effect that the gluon mass has on the ACM is nearly independent of the mass mechanism, at least for the small gluon mass that we have considered.

Therefore, we can conclude that the ACM of the light quarks have significant, and possibly observable, dependence on gluon mass, irrespective of how the mass is generated. As higher energies and luminosities become accessible to the LHC, precision measurements of the ACM of quarks should become possible. While a measurement of the top quark ACM is unlikely to constrain the gluon mass, data for other quarks will be able to put bounds on the mass of the gluon. 


\section{References}

[1] J. M. Cornwall, Phys. Rev. D 26, 1453 (1982).

[2] A. C. Aguilar and J. Papavassiliou, Phys. Rev. D 81, 034003 (2010).

[3] G. Parisi and R. Petronzio, Phys. Lett. B 94, 51 (1980).

[4] J. H. Field, Phys. Rev. D 66, 013013 (2002).

[5] F. J. Yndurain, Phys. Lett. B 345, 524 (1995).

[6] S. Nussinov and R. Shrock, Phys. Rev. D 82, 034031 (2010).

[7] G. Curci and R. Ferrari, Nuovo Cim. A 32, 151 (1976).

[8] T. J. Allen, M. J. Bowick and A. Lahiri, Mod. Phys. Lett. A 6, 559 (1991).

[9] D. S. Hwang and C. Y. Lee, J. Math. Phys. 38, 30 (1997) [hep-th/9512216].

[10] A. Lahiri, Phys. Rev. D 55, 5045 (1997) [hep-ph/9609510].

[11] CMS Collaboration [CMS Collaboration], Search for Anomalous Top Chromomagnetic Dipole

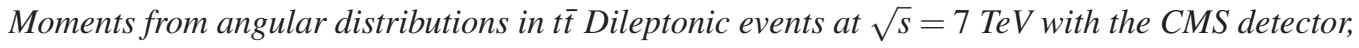
CMS-PAS-TOP-14-005 (2014).

[12] A. Lahiri and D. Mukhopadhyay, Unitarity in $W W \rightarrow W W$ elastic scattering in topologically massive SU(2) gauge theory, arXiv:1107.1501 [hep-ph].

[13] I. D. Choudhury and A. Lahiri, Mod. Phys. Lett. A 30, 1550113 (2015) [arXiv:1409.0073 [hep-ph]].

[14] J. Beringer et al. [Particle Data Group Collaboration], Review of Particle Physics (RPP), Phys. Rev. D 86, 010001 (2012) and 2013 partial update for the 2014 edition. . 